\title{
Propriétés cinétiques et thérapeutiques des lactones macrocycliques endectocides, doramectine et moxidectine, chez le bovin N'Dama (Bos taurus) au Gabon
}

\author{
T. Bengone-Ndong ${ }^{1,2} *$ E. Adjomo-Mba ${ }^{1}$ \\ M.-N. Mabika-Moussavou ${ }^{3}$ F. Meza ${ }^{1}$ \\ M. Kombila ${ }^{3}$ M. Alvinerie ${ }^{4}$
}

Mots-clés

Bovin N'Dama - Anthelminthique Pharmacocinétique - Gabon.

\begin{abstract}
Résumé
La cinétique plasmatique et l'efficacité de la doramectine et de la moxidectine ont été étudiées chez le bovin de race taurine N'Dama après administration sous-cutanée à la dose recommandée pour les bovins $(0,2 \mathrm{mg} / \mathrm{kg})$. Les valeurs du temps de demi-absorption ont montré une absorption significativement plus rapide de la moxidectine $(0,05$ jour pour la moxidectine contre 1,7 jour pour la doramectine). Les concentrations maximales obtenues n'ont pas été statistiquement différentes, mais elles ont été atteintes plus rapidement avec la moxidectine $(0,3$ jour) qu'avec la doramectine (4,8 jours). L'exposition, mesurée par l'aire sous la courbe, a été plus importante pour la doramectine (458 ng.jour/ml) que pour la moxidectine (159 ng.jour/ml), alors que la moxidectine a semblé plus rémanente. Les deux molécules ont présenté une efficacité de 100 p. 100 entre les quinzième et trentième jours qui ont suivi le traitement. Ces résultats sont comparables à ceux obtenus chez les autres races taurines de bovins. La doramectine et la moxidectine peuvent donc être recommandées dans la lutte contre les parasitoses gastro-intestinales de la vache $\mathrm{N}^{\prime}$ Dama en région tropicale humide.
\end{abstract}

\section{INTRODUCTION}

Les maladies parasitaires et les contraintes alimentaires constituent les principaux facteurs responsables de la faible productivité des bovins en Afrique tropicale, et la lutte contre les maladies parasitaires reste un des défis à relever. Pour lutter contre ces maladies,

\footnotetext{
1. Institut de recherches agronomiques et forestières, Centre national de la recherche scientifique et technologique, BP 2246 Libreville, Gabon.

2. Ecole normale supérieure, Libreville, Gabon.

3. Département de parasitologie-mycologie et de médecine tropicale, Université des sciences de la santé, Libreville, Gabon.

4. Laboratoire de pharmacologie - toxicologie, Inra, Toulouse, France.

* Auteur pour la correspondance

Tél.: +2417308 59; fax : +241732578

E-mail : tbengone@refer.ga ; bengonendong@yahoo.fr
}

de nombreux produits sont commercialisés sur le marché africain. Les macrolides endectocides constituent la classe thérapeutique la plus largement utilisée en raison tant de leur spectre d'action que de leur efficacité remarquable contre des parasites divers $(19,20)$.

Dans un contexte où l'élevage moderne est de plus en plus confronté aux problèmes liés aux résidus (sécurité alimentaire) et au développement de la résistance aux anthelminthiques, l'usage d'endectocides doit se faire de façon raisonnée. Il est en effet nécessaire de choisir les doses adaptées, les moments les plus adéquats pour les traitements, et de définir la durée de ces traitements, leur répétition et les animaux cibles. Il convient ainsi de donner aux antiparasitaires endectocides les meilleures conditions pour exercer leur action en milieu tropical africain en prenant en compte notamment les particularités des races locales et les contraintes environnementales. 
L'activité anthelminthique des endectocides est liée à la fois à l'action spécifique du médicament sur le parasite, avec une paralysie par interférence au niveau de la transmission des signaux nerveux contrôlés par le glutamate (17), et à la présence effective du pharmacophore sur le site d'action en termes de concentration et de durée $(2,10)$. Par ailleurs, il est maintenant généralement admis que l'effet antiparasitaire est plus étroitement lié au niveau d'exposition mesuré chez l'animal qu'à la dose administrée (18). En effet, la dose est une variable imposée, transformée par l'organisme en un profil de concentration via les propriétés physiologiques et métaboliques de l'animal (biodisponibilité, distribution, clairance). Dès lors, le profil des concentrations plasmatiques est une variable explicative beaucoup plus pertinente que la dose.

Des études récentes ont permis de mettre en évidence des différences importantes en termes de biodisponibilité de l'ivermectine $(4,5)$ et de l'éprinomectine $(4,6)$ entre le zébu Gobra du Sénégal et les bovins européens de type taurin. Ces différences sont vraisemblablement la conséquence de différences physiologiques et métaboliques entre les races et les espèces (Bos taurus et Bos indicus). Elles sont de nature à affecter les propriétés pharmacologiques des endectocides lorsque ces médicaments sont administrés aux doses retenues pour les bovins.

Cette étude de la doramectine et de la moxidectine chez le bovin N'Dama a eu pour objectif, d'une part, de déterminer les niveaux des concentrations plasmatiques et les paramètres pharmacocinétiques après administration sous-cutanée à la dose recommandée pour les bovins $(0,2 \mathrm{mg} / \mathrm{kg})$, et, d'autre part, d'évaluer l'efficacité de ces deux formulations dans la lutte contre le parasitisme interne (strongles) en milieu équatorial humide.

\section{MATERIEL ET METHODES}

\section{Cadre d'étude}

L'étude a été réalisée d'avril à octobre 2006 au ranch Biendi, situé à $14 \mathrm{~km}$ de la ville de Mouila dans la province de la Ngounié au Gabon. Dans cette province le climat est de type équatorial ; la pluviométrie est inégalement répartie le long de l'année, variant de 1500 à $3000 \mathrm{~mm}$ par an ; les températures moyennes annuelles sont d'environ $25^{\circ} \mathrm{C}$.

\section{Installations et matériel d'élevage}

Les installations d'élevage comprenaient deux compartiments, l'un réservé aux soins et l'autre au logement des bovins. L'enclos était équipé d'un abreuvoir, d'un couloir de contention, et de cordes permettant d'immobiliser les animaux lors des traitements et des soins. Un accès libre au pâturage a été soigneusement aménagé pendant toute la période de l'étude.

\section{Animaux}

L'étude a porté sur 14 bovins de race N'Dama. Il s'agissait de femelles âgées de 5 à 6 ans dont le poids était compris entre 138 et $243 \mathrm{~kg}$. Elles ont été identifiées par marquage sur le flanc et réparties en deux lots de sept animaux homogènes. Les animaux du premier lot (poids moyen de 194,8 \pm 19,3 kg) ont reçu la doramectine et ceux du deuxième lot (poids moyen de 202,4 $\pm 18,7 \mathrm{~kg}$ ) la moxidectine.

\section{Formulations et traitement des animaux}

La Cydectine ${ }^{\circledR}$ et le Dectomax ${ }^{\circledR}$, solutions injectables à base de moxidectine pour la première et de doramectine pour le second ont été utilisés. La Cydectine est distribuée sous forme de solution aqueuse à 1 p. 100 par Fort Dodge Santé animale (Tours, France). Le Dectomax a été fourni par le laboratoire Pfizer (Pocé-sur-Cisse, France) sous forme d'une solution huileuse à 1 p. 100. La Cydectine et le Dectomax ont été administrés aux animaux par injection sous-cutanée d'une dose unique de $0,2 \mathrm{mg} / \mathrm{kg}$, dose recommandée pour le traitement des bovins.

\section{Prélèvements}

Des prises de sang ont été effectuées chez les animaux traités par ponction de la veine jugulaire, et le sang a été recueilli dans des tubes contenant de l'héparinate de lithium comme anticoagulant à $0,30 \min , 1,2,4,8,12,24$ et 36 heures, puis à 2, 3, 4, 6, 8, 10, $15,20,25,30$ et 40 jours après le traitement sur l'ensemble des animaux. Les échantillons sanguins ont été centrifugés et le plasma conservé à $-18^{\circ} \mathrm{C}$ jusqu'à l'analyse. Des prélèvements de matières fécales destinées aux études parasitologiques ont été effectués trois jours avant le traitement puis à $\mathrm{J}_{0}, \mathrm{~J}_{7}, \mathrm{~J}_{14}, \mathrm{~J}_{21}, \mathrm{~J}_{28}, \mathrm{~J}_{35}$ et $\mathrm{J}_{40}$. Ils ont été conditionnés dans du formol à 10 p. 100 .

\section{Détermination des concentrations plasmatiques}

La détermination des concentrations plasmatiques de doramectine et de moxidectine a été réalisée grâce à une technique de chromatographie liquide haute performance (HPLC) après formation d'un dérivé fluorescent, selon le protocole décrit par de Montigny et coll. (8), et modifié par Alvinerie et coll. (1).

Pour chaque échantillon, $0,75 \mathrm{ml}$ d'acétonitrile et $0,25 \mathrm{ml}$ d'eau désionisée ont été ajoutés à $1 \mathrm{ml}$ de plasma dans un tube Eppendorf de $2 \mathrm{ml}$. Après $20 \mathrm{~min}$ d'agitation, les tubes ont été centrifugés pendant 4 min à 13000 tours $/ \mathrm{min}$ et à $4{ }^{\circ} \mathrm{C}$. Les surnageants ont ensuite été déposés sur une colonne d'extraction en phase solide (Supelclean C18, $100 \mathrm{mg}$ ) et élués avec $1 \mathrm{ml}$ de méthanol, après conditionnement et rinçage de la colonne, successivement par $2 \mathrm{ml}$ d'eau et $2 \mathrm{ml}$ d'un mélange eau-méthanol (75:25). L'éluat a été évaporé à $60{ }^{\circ} \mathrm{C}$ sous azote, puis ont été ajoutés successivement $100 \mathrm{ml}$ d'un mélange de $\mathrm{N}$-méthylimidazole-acétonitrile (1:1) et $150 \mathrm{ml}$ d'un mélange d'anhydride trifluoroacétique-acétonitrile (1:2). Après la réaction de fluorescence (30 s), une fraction aliquote a été injectée dans le chromatographe.

La phase mobile a été composée d'un mélange de trois solvants : acide acétique à 0,2 p. 100, méthanol et acétonitrile (4:40:56). Ils ont été injectés à travers la colonne à un débit égal à $1,5 \mathrm{ml} / \mathrm{min}$. La détection de la fluorescence a été effectuée à une longueur d'onde d'excitation de $355 \mathrm{~nm}$ et une longueur d'onde d'émission de $465 \mathrm{~nm}$.

Le dosage de chaque cinétique s'est effectué sur la base d'une courbe d'étalonnage comprenant les concentrations suivantes : $0 ; 0,5 ; 1 ; 2,5 ; 5 ; 10 ; 25$; et $50 \mathrm{ng} / \mathrm{ml}$. La limite de quantification de la méthode a été établie à $0,10 \mathrm{ng} / \mathrm{ml}$ pour les deux molécules. Le coefficient de variation mesuré sur les points dosés en duplicata a été de 4,4 p. 100 pour la doramectine et de 4,6 p. 100 pour la moxidectine.

\section{Analyse pharmacocinétique}

Les concentrations plasmatiques de doramectine et de moxidectine ont fait l'objet d'une analyse pharmacocinétique à l'aide d'un modèle bicompartimental en tenant compte du profil de concentrations grâce au programme informatique Kinetica, version 4.2 (InnaPhase, Philadelphie, Etats-Unis). Ce modèle est décrit par l'équation triexponentielle :

$C(t)=A_{1} e^{-\alpha t}+A_{2} e^{-\beta t}-A_{3} e^{-k a t}$ 
où $\mathrm{A}_{1}, \mathrm{~A}_{2}$ et $\mathrm{A}_{3}$ sont les interceptions, $\mathrm{C}$ la concentration plasmatique à un temps $\mathrm{t}$, ka la constante d'absorption, $\alpha$ la constante de distribution et $\beta$ la constante d'élimination $(11,23,24)$.

Les temps de demi-vie des phases d'absorption $\left(\mathrm{t}_{1 / 2 \mathrm{ab}}\right)$ et d'élimination $\left(\mathrm{t}_{1 / 2 \mathrm{el}}\right)$ correspondaient respectivement à $\ln 2 / \mathrm{ka}$ et $\ln 2 / \beta$. L'aire sous la courbe des concentrations en fonction du temps (area under the curve, AUC) et le temps moyen de résidence (mean residence time, MRT) ont été calculés en utilisant la méthode des trapèzes. La concentration maximale $\left(\mathrm{C}_{\max }\right)$ et le temps nécessaire pour atteindre cette concentration $\left(\mathrm{t}_{\max }\right)$ ont été déterminés directement à partir du profil de concentrations.

\section{Analyse statistique}

L'analyse statistique a été effectuée au moyen du logiciel Statview (version 5.0). Le test de Kolmogorov-Smirnov a été utilisé pour vérifier la distribution normale des données. Un test $t$ de Student a été réalisé pour déterminer les différences et préciser les degrés de signification pour chaque paramètre pharmacocinétique entre la doramectine et la moxidectine ; le seuil de signification a été limité à une probabilité inférieure ou égale à 5 p. 100.

\section{Efficacité thérapeutique}

L'efficacité thérapeutique des deux produits mesurée par l'excrétion fécale des œufs de nématodes [exprimée en nombre d'œufs par gramme (OPG) de matières fécales] a été déterminée par la méthode de McMaster décrite par Thienpont et coll. (21).

Deux grammes de matières fécales conservées dans une solution formolisée à 10 p. 100 ont été mis en suspension dans $60 \mathrm{ml}$ de solution saturée de $\mathrm{NaCl}$. Pour se débarrasser des grosses particules, la solution a été tamisée et la suspension remuée soigneusement pour obtenir un mélange homogène. La cellule de comptage a ensuite été remplie, légèrement inclinée pour permettre aux bulles d'air de s'échapper, à l'aide d'une pipette Pasteur. Après quelques minutes, les œufs flottant à la surface du liquide d'enrichissement ont adhéré à la lame couvrant la cellule. Ils ont alors été facilement comptés à faible grossissement.

Les cellules utilisées au cours de cet essai comportaient deux cavités. Chacune d'elle avait une surface de 10 x $10 \mathrm{~mm}$. L'espace entre la cellule et sa lamelle de couverture était de $1,5 \mathrm{~mm}$. Chaque cavité contenait donc $0,15 \mathrm{ml}$ de liquide. Pour chaque contrôle coproscopique et pour chaque échantillon, deux cavités ont été prises en compte (soit $0,30 \mathrm{ml}$ ). Après comptage des œufs, la détermination des OPG s'est faite selon la formule suivante :

$\mathrm{OPG}=\mathrm{X} * 103$

Avec :

$103=62 / 2 * 1 / 0,30 ;$

$62 \mathrm{ml}=$ volume du mélange homogène $(60 \mathrm{ml}$ de la solution saturée de $\mathrm{NaCl}+2 \mathrm{~g}$ de matières fécales);

2 = quantité de matières fécales $(\mathrm{g})$;

$0,30 \mathrm{ml}=$ volume des deux cavités prises en compte dans l'évaluation ;

$\mathrm{X}=$ nombre d'œufs trouvés dans $0,30 \mathrm{ml}$.

Dans cette évaluation de l'efficacité thérapeutique, les valeurs des OPG obtenues avant le traitement des animaux ont été considérées comme témoins. Un test $U$ non paramétrique de Wilcoxon-MannWhitney a été réalisé pour déterminer les différences d'efficacité entre la doramectine et la moxidectine.

\section{RESULTATS}

\section{Pharmacocinétique}

La figure 1 représente l'évolution des concentrations plasmatiques de doramectine et de moxidectine après administration sous-cutanée de 0,2 mg par kilogramme de poids vif. Les valeurs moyennes des paramètres pharmacocinétiques ainsi que les résultats du test $t$ de Student sont présentés dans le tableau I. L'examen de ces paramètres a montré une absorption significativement plus rapide de la moxidectine, indiquée par les valeurs du temps de demi-absorption. Les concentrations maximales obtenues n'ont pas été statistiquement différentes, mais cette concentration maximale a été atteinte plus rapidement avec la moxidectine qu'avec la doramectine. L'aire sous la courbe des concentrations en fonction du temps a été plus importante pour la doramectine que pour la moxidectine, alors que la moxidectine a eu un temps moyen de résidence plus important.

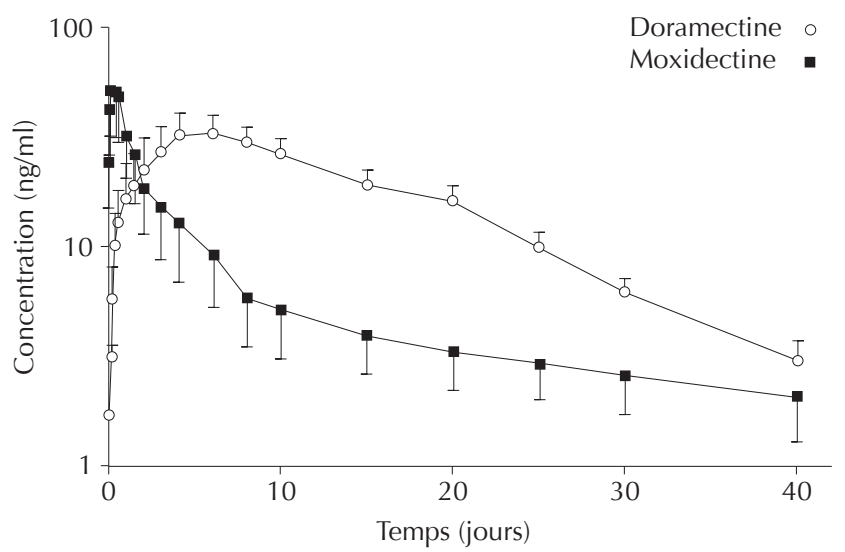

Figure 1 : évolution des concentrations plasmatiques de doramectine et de moxidectine chez sept bovins N'Dama après administration sous-cutanée d'une dose unique de $0,2 \mathrm{mg} / \mathrm{kg}$ (valeurs moyennes \pm écarts-types).

\section{Tableau I}

Valeurs pharmacocinétiques moyennes de la doramectine et de la moxidectine chez sept bovins N'Dama après administration sous-cutanée $\mathrm{d}^{\prime}$ une dose unique de $0,2 \mathrm{mg} / \mathrm{kg}$

\begin{tabular}{|c|c|c|c|c|c|}
\hline Paramètre $\Lambda$ & Moxidectine & E.T. & Doramectir & e E.T. & $\mathbf{P}$ \\
\hline$t^{1} 1 / 2 a b$ (jours) & 0,05 & 0,02 & 1,70 & 1,18 & 0,014 * \\
\hline $\mathrm{C}_{\max }(\mathrm{ng} / \mathrm{ml})$ & 40,9 & 8,1 & 34,9 & 6,6 & $0,24 \mathrm{NS}$ \\
\hline$t_{\max }$ (jours) & 0,3 & 0,1 & 4,8 & 1,1 & $<0,0001^{*}$ \\
\hline$t^{1 / 2}$ el (jours) & 13,1 & 2,1 & 6,3 & 1,4 & $0,0003 *$ \\
\hline$A \cup C$ (ng.j/ml) & l) 159 & 35 & 458 & 61 & $<0,0001^{*}$ \\
\hline MRT (jours) & 14,1 & 3,3 & 11,2 & 1,1 & $0,11 \mathrm{NS}$ \\
\hline
\end{tabular}

\section{E.T. : écart-type}

$\mathrm{t} \frac{1}{2} \mathrm{ab}$ : temps de demi-vie des phases d'absorption

$\mathrm{C}_{\max }$ : concentration maximale

$\mathrm{t}_{\max }$ : temps pour concentration maximale

$\mathrm{t} 1 / 2$ el : temps de demi-vie des phases d'élimination

AUC : aire sous la courbe

MRT : temps moyen de résidence

* Différences significatives $(\mathrm{p}<0,05)$

NS : différences non significatives $(p>0,05)$ 


\section{Efficacité thérapeutique}

Les résultats de l'efficacité thérapeutique des deux molécules, en termes d'évolution du nombre d'OPG de strongles après le traitement, sont présentés dans le tableau II. Ces résultats ont montré une bonne protection des animaux pendant la durée de l'expérimentation avec la doramectine aussi bien qu'avec la moxidectine. Les différences observées entre les deux endectocides n'étaient pas statistiquement significatives.

\section{- DISCUSSION}

La moxidectine et la doramectine ont été détectées chez tous les animaux pendant toute la durée de l'étude (40 jours). La technique de dosage utilisée, au niveau de l'extraction, de la dérivatisation et de la chromatographie, a été validée. Le pourcentage d'extraction moyen a été établi à 95 p. 100 et la limite de quantification à $0,10 \mathrm{ng} / \mathrm{ml}$ pour les deux molécules. Le coefficient de variation mesuré sur les points dosés en duplicata a été de 4,4 p. 100 pour la doramectine et de 4,6 p. 100 pour la moxidectine. Les coefficients de corrélation ont été respectivement de 0,990 et 0,999 dans l'intervalle de concentrations allant de 0,50 à $50 \mathrm{ng} / \mathrm{ml}$.

L'analyse des paramètres pharmacocinétiques a montré une grande différence entre la moxidectine et la doramectine chez le N'Dama lorsque ces molécules étaient administrées par voie sous-cutanée. L'aire sous la courbe obtenue avec la moxidectine a été trois fois inférieure à celle obtenue dans les mêmes conditions avec la doramectine. La biodisponibilité de la doramectine a donc été supérieure à celle de la moxidectine chez la vache N'Dama. La comparaison des paramètres d'absorption a montré une absorption plus rapide de la moxidectine, alors que les paramètres d'élimination ont montré une rémanence plus importante de la moxidectine par rapport à la doramectine.

Ces différences ont été observées par de nombreux auteurs chez la plupart des autres espèces animales $(3,9,12)$. Elles peuvent être expliquées par les propriétés physico-chimiques propres à chaque principe parental, associées à la nature de la formulation. Il a notamment été montré que la cinétique des endectocides est largement influencée par la formulation $(16,22)$. Ainsi, la comparaison du profil pharmacocinétique des trois endectocides majeurs (ivermectine, doramectine, moxidectine), administrés par voie sous-cutanée dans le cadre d'une étude standardisée, a révélé un profil similaire pour l'ivermectine et la doramectine, tandis que la moxidectine se caractérise par un processus d'absorption plus rapide et une rémanence plus longue (12), résultant d'une formulation différente (aqueuse) et d'un stockage plus intense au niveau graisseux.

Cet effet de la formulation sur le processus d'absorption a été longuement documenté tant pour l'ivermectine (16) que pour la doramectine (22). Cela a justifié le choix d'une formulation huileuse par Pfizer (doramectine) et Fort-Dodge (moxidectine), cette dernière permettant de ne pas majorer l'importante rémanence résultant du stockage au niveau de la graisse.

\section{Tableau II}

Evolution de la quantité moyenne du nombre d'œufs par gramme de matières fécales chez des bovins N'Dama traités à la doramectine et à la moxidectine à la dose unique de 0,2 mg/kg de poids vif

\begin{tabular}{|c|c|c|c|c|c|c|c|c|}
\hline \multirow[b]{2}{*}{ Traitement } & \multicolumn{7}{|c|}{ Nombre d'œufs par gramme de matières fécales } & \multirow[b]{2}{*}{$J_{40}$} \\
\hline & Animaux & $\mathrm{J}_{-3}$ & $\mathbf{J}_{0}$ & $\mathbf{J}_{8}$ & $\mathrm{~J}_{15}$ & $\mathbf{J}_{22}$ & $\mathbf{J}_{35}$ & \\
\hline \multirow[t]{8}{*}{ Doramectine } & 1 & 309 & 309 & 103 & 0 & 0 & 0 & 0 \\
\hline & 2 & 618 & 551 & 206 & 103 & 0 & 0 & 0 \\
\hline & 3 & 309 & 206 & 103 & 0 & 0 & 0 & 0 \\
\hline & 4 & 206 & 206 & 103 & 0 & 0 & 0 & 0 \\
\hline & 5 & 206 & 206 & 103 & 0 & 0 & 0 & 103 \\
\hline & 6 & 309 & 309 & 103 & 0 & 0 & 0 & 0 \\
\hline & 7 & 206 & 206 & 103 & 0 & 0 & 0 & 0 \\
\hline & Moyenne & 309 & 280 & 118 & 15 & 0 & 0 & 15 \\
\hline \multirow[t]{8}{*}{ Moxidectine } & 8 & 206 & 206 & 103 & 0 & 0 & 0 & 0 \\
\hline & 9 & 206 & 206 & 103 & 0 & 0 & 0 & 103 \\
\hline & 10 & 206 & 103 & 0 & 0 & 0 & 0 & 0 \\
\hline & 11 & 309 & 309 & 103 & 0 & 0 & 0 & 103 \\
\hline & 12 & 206 & 103 & 103 & 0 & 0 & 0 & 0 \\
\hline & 13 & 309 & 309 & 103 & 0 & 0 & 0 & 0 \\
\hline & 14 & 309 & 309 & 103 & 0 & 0 & 0 & 0 \\
\hline & Moyenne & 250 & 220 & 88 & 0 & 0 & 0 & 29 \\
\hline \multirow[t]{2}{*}{ Test $U$ (valeur de P) } & & 0,52 & 0,48 & 0,41 & 0,65 & $>0,99$ & $>0,99$ & 0,65 \\
\hline & & NS & NS & NS & NS & NS & NS & NS \\
\hline
\end{tabular}

NS : différences non significatives entre les deux lots $(p>0,05)$ 
Le rôle de la pharmacocinétique dans l'estimation de l'efficacité des endectocides repose sur l'hypothèse selon laquelle le profil plasmatique reflète le profil de concentration du principe actif au niveau du site d'action et est vraisemblablement à la base de l'efficacité des endectocides. De travaux réalisés chez les bovins avec la moxidectine (14) et l'ivermectine (15) montrent l'étroite corrélation qui relie les concentrations plasmatiques aux concentrations observées au niveau de sites d'action tels que la peau ou le mucus abomasal. En effet, non seulement les profils d'évolution sont parallèles, mais, de plus, les concentrations dans les tissus cibles sont supérieures à celles observées dans le plasma. Dès lors, on peut considérer que, dans le cas des endectocides, les paramètres plasmatiques sont des paramètres pertinents pour prédire l'activité anthelminthique au niveau du site d'action.

Les résultats de la présente étude sont comparables à ceux qui ont été obtenus chez les taurins $(12,13)$ et le zébu Gobra (7), en termes de biodisponibilité et de rémanence de la moxidectine et de la doramectine. Ils montrent que ces molécules peuvent être utilisées avantageusement dans la lutte contre les nématodes gastrointestinaux chez le N'Dama.

Concernant l'efficacité thérapeutique, l'évolution du nombre d'œufs de nématodes par gramme de matières fécales montre une réduction totale de l'excrétion des œufs de strongles à partir du quinzième jour après le traitement dans les deux cas. Cette efficacité a persisté pendant toute la durée de la période d'étude (40 jours). Ce résultat est en accord avec celui obtenu par l'étude de la pharmacocinétique qui montre que 40 jours après le traitement, les concentrations plasmatiques ont été nettement supérieures aux valeurs thérapeutiques efficaces $(0,2 \mathrm{ng} / \mathrm{ml})$. Toutefois, pour une meilleure appréciation de l'efficacité, un diagnostic post mortem avec comptage des vers adultes aurait été nécessaire afin de mieux évaluer le spectre d'activité. De plus, l'étude a été réalisée pendant la saison sèche, peu favorable à la pullulation des parasites. On peut donc penser que les faibles dénombrements d'œufs observés proviennent non seulement de l'efficacité des molécules employées, mais également d'une faible exposition aux parasites. Une étude réalisée pendant la période pluvieuse serait nécessaire.

\section{Remerciements}

Cette étude a été financée par la Fondation internationale pour la Science. Nous remercions particulièrement Mmes Ingrid Leemans (coordinatrice du programme scientifique), Annika Eriksson et Josiane Lindberg pour leurs encouragements. Nous remercions également M. et Mme Mangaga Moussavou qui nous ont ouvert les portes du ranch Biendi ; leur disponibilité a grandement facilité ce travail.

\section{BIBLIOGRAPHIE}

1. ALVINERIE M., SUTRA J.F., BADRI M., GALTIER P., 1995. Determination of moxidectin in plasma by high performance chromatography with fluorescence detection. J. Chromatogr. B, 674: 119124.

2. BAGGOT J.D., MCKELLAR Q.A., 1994. The absorption, distribution and elimination of anthelminthic drugs: the role of pharmacokinetics. J. vet. Pharmacol. Ther., 17: 409-419.

3. BARBER S., BOWLES V., LESPINE A., ALVINERIE M., 2003. The comparative serum disposition kinetics of subcutaneous administration of doramectin, ivermectin and moxidectin in the Australian Merino sheep. J. vet. Pharmacol. Ther., 26: 1-6.

4. BENGONE-NDONG T., ALVINERIE M., 2004. Macrolides antiparasitaires : propriétés pharmacologiques générales et recommandations d'usage dans le contexte vétérinaire africain. Revue Elev. Méd. vét. Pays trop., 57 : 49-58.
5. BENGONE-NDONG T., KANE Y., BA M.A., SANE I., SUTRA J.F., ALVINERIE M., 2005. Pharmacokinetics of ivermectin in zebu Gobra (Bos indicus). Vet. Parasitol., 128: 169-174.

6. BENGONE-NDONG T., KANE Y., BA M.A., SANE I., SUTRA J.F., ALVINERIE M., 2006. Eprinomectin in dairy zebu Gobra cattle (Bos indicus): plasma kinetics and excretion in milk. Parasitol. Res., 98: 501 506.

7. BENGONE-NDONG T., KIKI MVOUAKA S., KANE Y., LOUDY MOUKEDE W.A., PANGUY L.J., ALVINERIE M., 2008. A comparative kinetic study of doramectin and moxidectin in zebu Gobra cattle (Bos indicus). Parasitol. Res., 103: 1465-1467.

8. DE MONTIGNY P., SHIM J.S.K., PIVNICHNY J.V., 1990. Liquid chromatographic determination of ivermectin in animal plasma with trifluoroacetic anhydride and $\mathrm{N}$-methylimidazole as the derivatization reagent. J. Pharm. Biomed. Anal., 8: 507-511.

9. ESCUDERO E., CARCELES C.M., DIAZ M.S., SUTRA J.F., GALTIER P., ALVINERIE M., 1999. Pharmacokinetics of moxidectin and doramectin in goats. Res. vet. Sci., 67: 177-181.

10. GEARY T.G., SANGSTER N.C., THOMPSON D.P., 1999. Frontiers in anthelminthic pharmacology. Vet. Parasitol., 84: 275-295.

11. GIBALDI M., PERRIER D., 1982. Pharmacokinetics. New York, USA, Marcel and Dekker, p. 445-449.

12. LANUSSE C., LIFSCHITZ A., VIRKEL G., ALVAREZ L., SANCHEZ S., SUTRA J.F., GALTIER P., ALVINERIE M., 1997. Comparative plasma disposition kinetics of ivermectin, moxidectin and doramectin in cattle. J. vet. Pharmacol. Ther., 20: 91-99.

13. LANUSSE C., PRICHARD R., 1993. Relationship between pharmacological properties and clinical efficacy of ruminant anthelmintics. Vet. Parasitol., 49: 123-158.

14. LIFSCHITZ A., VIRKEL G., IMPERIALE F., GALTIER P., LANUSSE C., ALVINERIE M., 1999. Moxidectin in cattle: correlation between plasma and target tissues disposition. J. vet. Pharmacol. Ther., 22: 266-273.

15. LIFSCHITZ A., VIRKEL G., SALLOVITZ G., SUTRA J.F., GALTIER P., ALVINERIE M., LANUSSE C., 2000. Comparative distribution of ivermectin and doramectin to parasite location tissues in cattle. Vet. Parasitol., 87: 327-338.

16. LO P.K., FINK D.W., WILLIAMS J.B., BLODINGER J., 1985. Pharmacokinetic studies of ivermectin: effect of formulation. Vet. Res. Commun., 9: 251-268.

17. MCKELLAR Q.A., BENCHAOUI H., 1996. Avermectins and milbenmycins. J. vet. Pharmacol. Ther., 19: 331-351.

18. ROWLAND M., TOZER T., 1995. Clinical pharmacokinetics, concepts and application. Baltimore, MD, USA, Williams and Wikins, p. 60

19. SOLL M.D., BENZ G.W., CARMICHAEL I.H., GROSS S.J., 1990. Efficacy of ivermectin delivered from an intraruminal sustained release bolus against natural infections of live African tick species on cattle. Vet. Parasitol., 37: 285-296.

20. TALABI A.O., OYEKUNLE M.A., MARTINS E.O., TALABI A.M., 2003. Comparative study of the efficacies of two ivermectin on acarina ticks of cattle. Afr. J. Livest. Ext., 2: 44-46.

21. THIENPONT D., ROCHETTE F., VANPARIJS O.F.J., 1979. Diagnosing helminthiasis by coprological examination. Beerse, Belgium, Janssen Research Foundation, p. 205.

22. WICKS S.R., KAYE B., WEATHERLEY A.J., SMITH D.G., 1993. Effect of formulation on the pharmacokinetics and efficacy of doramectin. Vet. Parasitol., 49: 17-26.

23. YAMAOKA K., NAKAGAWA T., UNO T., 1978. Application of Akaike's information criterion (AIC) in the evaluation of linear pharmacokinetic equations. J. Pharmacokinet. Biopharm., 6: 165-175.

24. YAMAOKA K., TANIGAWARA K., NAKAGAWA T., UNO T., 1981. A pharmacokinetic analysis program (MULTI) for microcomputer. /. Pharmacobiodyn., 4: 879-885.

Accepté le 30.05.2011 


\section{Summary}

Bengone-Ndong T., Adjomo-Mba E., Mabika-Moussavou M.-N., Meza F., Kombila M., Alvinerie M. Kinetic and therapeutic properties of macrocyclic lactone endectocides, doramectin and moxidectin, in N'Dama cattle (Bos taurus) in Gabon

The plasma kinetics and efficacy of doramectin and moxidectin were studied in N'Dama taurine cattle after subcutaneous administration at the recommended dose of $0.2 \mathrm{mg} / \mathrm{kg}$. Absorption half-life values showed that the absorption of moxidectin (0.05 day) was significantly faster than that of doramectin (1.7 day). No significant differences were observed in maximum concentrations, but they were reached earlier with moxidectin ( 0.3 day) than with doramectin (4.8 days). Exposure, measured by the area under the curve, was higher for doramectin (458 ng.day/ml) than for moxidectin $(159$ ng.day/ml), whereas moxidectin seemed more remanent. Both molecules showed $100 \%$ efficacy between days 15 and 30 posttreatment. These results are similar to those observed in other taurine breeds. Doramectin and moxidectin can therefore be recommended to control gastrointestinal parasitoses in N'Dama in humid tropical regions.

Keywords: N'Dama cattle - Anthelmintic - Pharmacokinetics - Gabon.

\section{Resumen}

Bengone-Ndong T., Adjomo-Mba E., Mabika-Moussavou M.-N., Meza F., Kombila M., Alvinerie M. Propiedades cinéticas y terapéuticas de los lactones macro cíclicos endectocidas, doramectina y moxidectina en el bovino N'Dama (Bos taurus) en Gabón

Se estudiaron la cinética plasmática y la eficacidad de la doramectina y de la moxidectina en el bovino de raza taurina N'Dama, después de la administración sub cutánea a la dosis recomendada para los bovinos $(0,2 \mathrm{mg} / \mathrm{kg})$. Los valores del tiempo de media absorción mostraron una absorción significativamente más rápida para moxidectina $(0,05$ día para la moxidectina contra 1,7 día para la doramectina). Las concentraciones máximas obtenidas no fueron significativamente diferentes, pero se alcanzaron más rápidamente con la moxidectina (0,3 día) que con la doramectina (4,8 días). La exposición, medida mediante el área bajo la curva, fue más importante para la doramectina (458 ng.día/ml) que para la moxidectina (159 ng.día/ml), mientras que la moxidectina pareció más remanente. Las dos moléculas presentaron una eficacidad de $100 \%$ entre los días quince y treinta que siguieron al tratamiento. Estos resultados son comparables a los obtenidos en las otras razas taurinas de bovinos. La doramectina y la moxidectina pueden así ser recomendadas en la lucha contra las parasitosis gastrointestinales de la vaca N’Dama en la región tropical húmeda.

Palabras clave : Bovino N'Dama - Antihelmíntico - Farmacocinética - Gabon 